\title{
ENHANCEMENT OF SNOWSCAT FOR TOMOGRAPHIC OBSERVATION CAPABILITIES
}

\author{
Othmar Frey $^{1,2}$, Charles L. Werner ${ }^{1}$, Martin Schneebeli ${ }^{3}$, Amy Macfarlane $^{3}$, and Andreas Wiesmann ${ }^{1}$ \\ ${ }^{1}$ Gamma Remote Sensing, CH-3073 Gümligen, Switzerland \\ ${ }^{2}$ Earth Observation \& Remote Sensing, ETH Zurich, Zurich, Switzerland \\ ${ }^{3}$ WSL Institute for Snow and Avalanche Research SLF, Davos, Switzerland \\ http://www.gamma-rs.ch
}

\begin{abstract}
The SnowScat device, a tower-mounted fully polarimetric scatterometer for measurements of the radar crosssection of snow at X-band up to Ku-band, has recently been enhanced to also support a tomographic profiling mode. The new tomographic profiling capability of SnowScat allows for performing high-resolution observations providing further insights into the complex electromagnetic interaction within snowpacks. In this paper, we present first results obtained from a series of tomographic profiles of a snowpack acquired with the enhanced SnowScat device at a test site of SLF in Davos, Switzerland, between Dec. 2014 and March 2015.
\end{abstract}

Key words: SnowScat, microwave remote sensing, snow, tomography, tomographic profiling, SAR tomography, scatterometer, X-band, Ku-band, ESA.

\section{INTRODUCTION}

The SnowScat instrument [1-4] has originally been designed as a tower-mounted fully polarimetric scatterometer for measurements of the radar cross-section of snow at X-band up to $\mathrm{Ku}$-band over a frequency range of 9.1517.9 GHz. Here, we present an extension of SnowScat for tomographic profiling capabilities. This extension aims at enhancing the SnowScat device in order to better respond to the ESAC recommendations made on the deselected $\mathrm{CoReH} 2 \mathrm{O}$ candidate following the User Consultation meeting in March 2013 for the 7 Earth Explorer mission. Such new capability allows for performing highresolution tomographic profiling observations providing further insights into the complex electromagnetic interaction within snowpacks.

Experiments of a similar kind (using different hardware and applying 2-D aperture synthesis in elevation and azimuth) have been reported in [5, 6] that already indicated the potential of tomographic observations of snowpacks using microwave remote sensing in the X-/Ku-band.
The tomographic measurement concept of the SnowScat device is different in the sense that aperture synthesis is only performed along the elevation direction (along the direction perpendicular to the line of sight). This means that the azimuth resolution is frequency-dependent and is governed by the beamwidth of the SnowScat antenna; therefore, the azimuth resolution decreases with increasing range distance. The reason behind this configuration is that the enhanced SnowScat device is designed to operate in both modes, the original scatterometer mode, and the new tomographic profiling mode. The user can switch between the two modes at his discretion.

In this paper, we present and discuss first results of a series of tomographic profiles of snowpacks acquired with the enhanced SnowScat device at a test site of SLF in Davos, Switzerland, between December 2014 and March 2015.

\section{METHODS}

\subsection{Tomographic measurement setup}

The nominal acquisition scenario that was planned and implemented at the test site in Davos, Switzerland, is shown in Fig. 1. The SnowScat device (see Table 1 for the system specifications) is attached to a rail on a triangular truss. The SnowScat device can be moved along this rail (tilted by 45 degree) within a maximal total synthetic aperture length of $2.22 \mathrm{~m}$. The centre of the synthetic aperture is located approximately $7.5 \mathrm{~m}$ above ground. At this central position the SnowScat device is pointing at the center of a tomographic test target, assuming an incidence angle of 45 degree. The tomographic test target is used as a reference and for validation purposes. It consists of an array of eight aluminium spheres mounted on a carbon tube. The dimensions of the spheres and the associated radar cross sections are given in Table 2 In Table 3 the parameters for the tomographic acquisition of the test campaign are shown. 


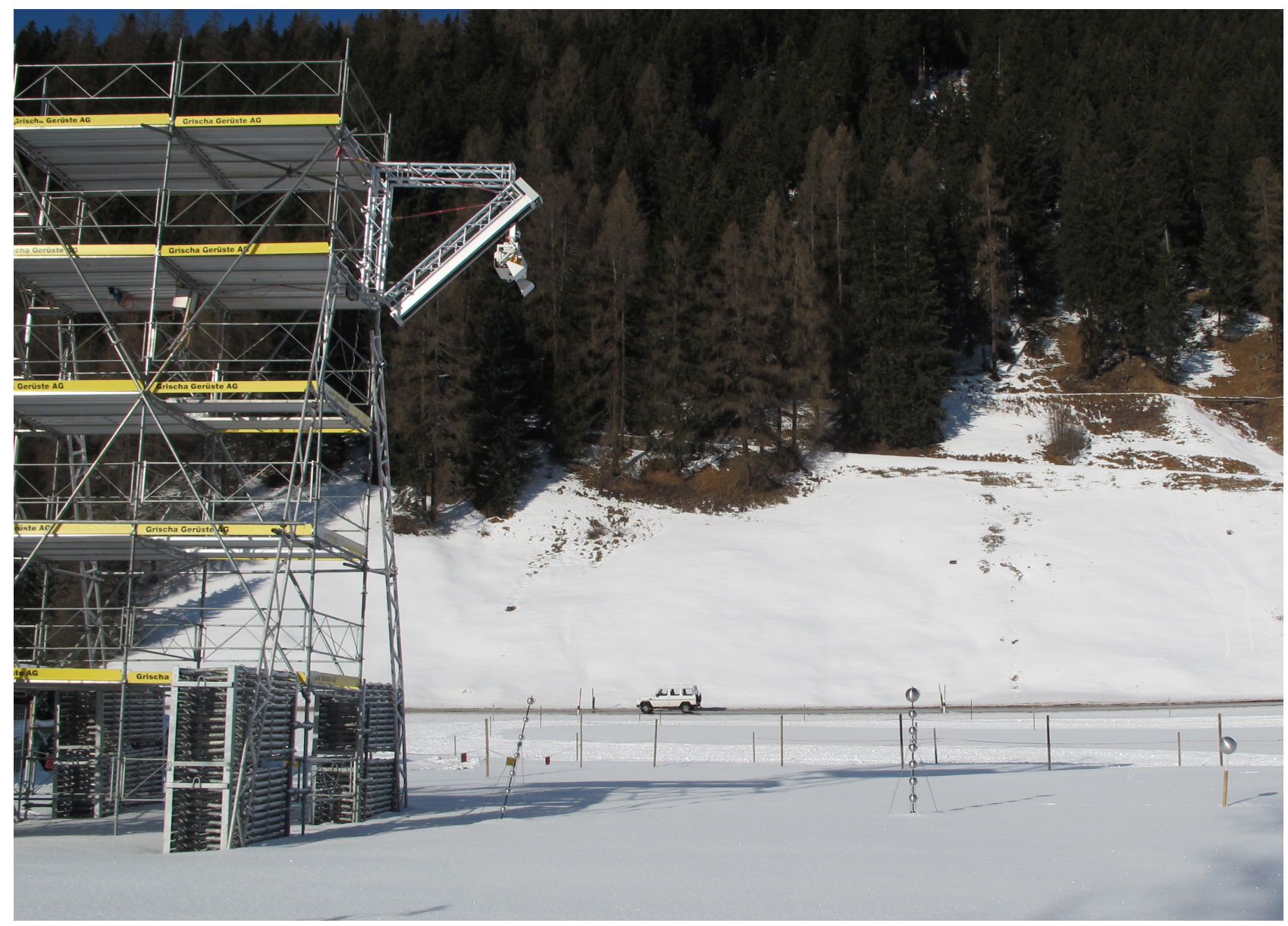

Figure 1. Tomographic measurement setup of the enhanced SnowScat device at the SLF test site in Davos, Switzerland. The SnowScat device is moveable along a tilted rail to create a synthetic aperture in elevation direction. In such a way, a high resolution is not only obtained in range direction but also in elevation direction (i.e., perpendicular to the mean line of sight). The situation shows the tomographic test target (in the center) made of 8 spheres on a carbon tube. (Close to the scaffolding another test target for the scatterometer mode and, on the far right, a calibration sphere are visible).

\subsection{Stripmap-mode resolution}

The SnowScat device can be moved along the rail by a total distance of $2.22 \mathrm{~m}$. Assuming an unconstrained elevation beamwidth a resolution of about $\delta_{n}=\lambda_{c} r_{0} /(2 L)=$ $0.05 \mathrm{~m}$ could be obtained at a range distance of $9 \mathrm{~m}$. However, since the SnowScat device was originally designed as a scatterometer the antenna beamwidth is rather narrow, between $4.6^{\circ}$ and $9.0^{\circ}$, depending on the frequency: $\theta_{b w}=\lambda / D$. So, at a range distance of approximately $r_{0}$ $=9 \mathrm{~m}$ the effective synthetic aperture for that location is only $L_{s t}=0.72 \mathrm{~m}$ at a beamwidth of $\theta_{b w}=4.6^{\circ}$. Therefore, the maximal resolution that can be expected in elevation direction (perpendicular to the line of sight) in stripmap mode is $\delta_{n_{s t}}=\lambda_{c} r_{0} /\left(2 L_{s t}\right)=0.15 \mathrm{~m}$. It is, however, possible to measure at different elevation angles while moving along the rail. This leads to a spotlight mode acquisition with a longer synthetic aperture approaching again a resolution of $0.05 \mathrm{~m}$ as obtained with the maximal synthetic aperture length allowed by the rail.

\subsection{Data processing}

The tomographic profiling is performed by coherently combining - in this case, time-domain back-projection (TDBP)-based aperture synthesis [7, 8] is applied-a number of SnowScat measurements taken at different antenna positions uniformly spaced along the tilted rail. The TDBP processing approach takes into account the actual 3-D geometry between the sensor positions and the illuminated volume while focusing the data. In particular, the TDBP algorithm implements a ray-tracing-like calculation of the wave propagation, which allows for including the refraction occurring at the air/snow interface and potential further refraction at different layers within the snowpack under inspection. A virtual range distance $R_{v}$ can be calculated iteratively based on the incidence angle $\theta$, the angle of refraction $\theta_{S}$, the refractive index $n_{s}$, and the different phase velocities $c$ (in air) and $v_{S}$ (in the snow volume). The delay-and-sum approach of the TDBP focusing can be written as (omitting the antenna 
Table 1. System specifications of SnowScat device

\begin{tabular}{ll}
\hline \hline Power & $230 \mathrm{~V}, \max \sim 60 \mathrm{~W}$ \\
Weight & $\sim 40 \mathrm{~kg}$ \\
Temperature range & $-40^{\circ} \mathrm{C}$ to $40^{\circ} \mathrm{C}$ \\
Antennas & Dual pol, $<10^{\circ}(3 \mathrm{~dB})$ \\
Antenna cross-pol & $<-25 \mathrm{~dB}$ \\
\hline Frequency & $\mathrm{SFCW}$ from 9.15 to $17.9 \mathrm{GHz}$ \\
Incidence angle & $-40^{\circ}$ to $110^{\circ}$ \\
Azimuth angle & $-180^{\circ}$ to $180^{\circ}$ \\
\hline Polarization & $\mathrm{HH}, \mathrm{HV}, \mathrm{VV}, \mathrm{VH}$ \\
Dynamic range & Receiver dynamic range $>80 \mathrm{~dB}$ with the $16 \mathrm{bit}$ ADC \\
Signal bias & $<0.5 \mathrm{~dB}$ \\
Gain characterization & Internal calibration, Calibration sphere (diameter: $25 \mathrm{~cm})$ \\
Control & Remote control through Ethernet, standalone \\
Data storage & Internal, external through Ethernet \\
RFI & Frequency blacklist \\
\hline \hline
\end{tabular}

gain pattern and the range-spreading loss):

$v\left(\vec{r}_{i}\right)=\sum_{k=1}^{M} g_{k}\left[R_{v}\left(\vec{r}_{i}, \vec{r}_{k}, n_{s}\right)\right] \cdot \exp \left[i 4 \pi / \lambda R_{v}\left(\vec{r}_{i}, \vec{r}_{k}, n_{s}\right)\right]$

where $\vec{r}_{i}$ is the 3-D position vector of the target location for which the tomographic inversion is performed, $\vec{r}_{k}$ is the $3-\mathrm{D}$ position vector of the antenna phase center at position $k$ within the synthetic aperture, $g_{k}(\ldots)$ is the rangecompressed signal at antenna position $k, \lambda$ is the wavelength of the carrier signal, $R_{v}\left(\vec{r}_{i}, \vec{r}_{k}, n_{s}\right)$ is the (virtual) range distance between antenna position $k$ and the location $\vec{r}_{i}$ taking into account the refraction, and $v\left(\vec{r}_{i}\right)$ is the tomographically focused signal at location $\vec{r}_{i}$. The range echo values at the correct distance are retrieved by a combined FFT-based and linear interpolation of the complexvalued echo. An antenna pattern weighting function in elevation is applied during the back-projection image formation. Multi-looking can then be performed by just spatially averaging the tomographic image. Note, that the tomographic slices (2-D plots in Fig. 2) are 1-look intensity images.

For the SnowScat measurement setup the geometry is well known. Therefore, one can assume that the following assumptions are valid as a first approximation:

1. antenna positions and position of the image reconstruction grid (area of interest) are known

2. a homogeneous snow layer with known depth is assumed

3. thus, the point of entry at the air/snow interface can be calculated based on the previous two assumptions.

The concept is based on calculating the "point of entry" (and its corresponding ray path) at the air/snow interface. Once the point of entry is known the incidence angles (which relate each point of the reconstruction grid to a specific measurement at a particular angle and antenna position), and the propagation time between antenna and grid point on the reconstruction grid can be computed from trigonometry.

Table 2. Radar cross sections (RCS) of spheres: $\sigma=\pi r^{2}$

\begin{tabular}{ll}
\hline \hline Radius of sphere $[\mathrm{m}]$ & $\mathbf{R C S ~}\left[\mathrm{m}^{2}\right]$ \\
\hline 0.10 & 0.0314 \\
0.075 & 0.0177 \\
0.06 & 0.0113 \\
0.05 & 0.0078 \\
\hline \hline
\end{tabular}

Table 3. Parameters for the tomographic acquisition of the test measurement

\begin{tabular}{ll}
\hline \hline Spacing between antenna positions & $0.04 \mathrm{~m}$ \\
Number of measurements along rail & 50 \\
Length. of synthetic aperture & $1.96 \mathrm{~m}$ \\
Elevation angle of antenna pointing direction & $45^{\circ}$ \\
\hline
\end{tabular}

\section{RESULTS}

In Fig. 2, a series of tomographic profiles obtained by TDBP-focusing of 50 range echoes of the $\mathrm{HH}$ polarization channel of the SnowScat device are given. Each 1-look image shows the situation at the test site in Davos, Switzerland, as shown in Fig. 2, the tomographic test target with its 8 aluminium spheres and the ground surface and/or the snowpack are imaged under snow-free and snow-covered condition. Aperture synthesis was performed by a TDBP approach taking into account a simple refraction model. Below each of the 2-D slices a vertical profile of mean intensity averaged over $1 \mathrm{~m}$ horizontally (see blue rectangle) is shown. In addition, two in-situ 


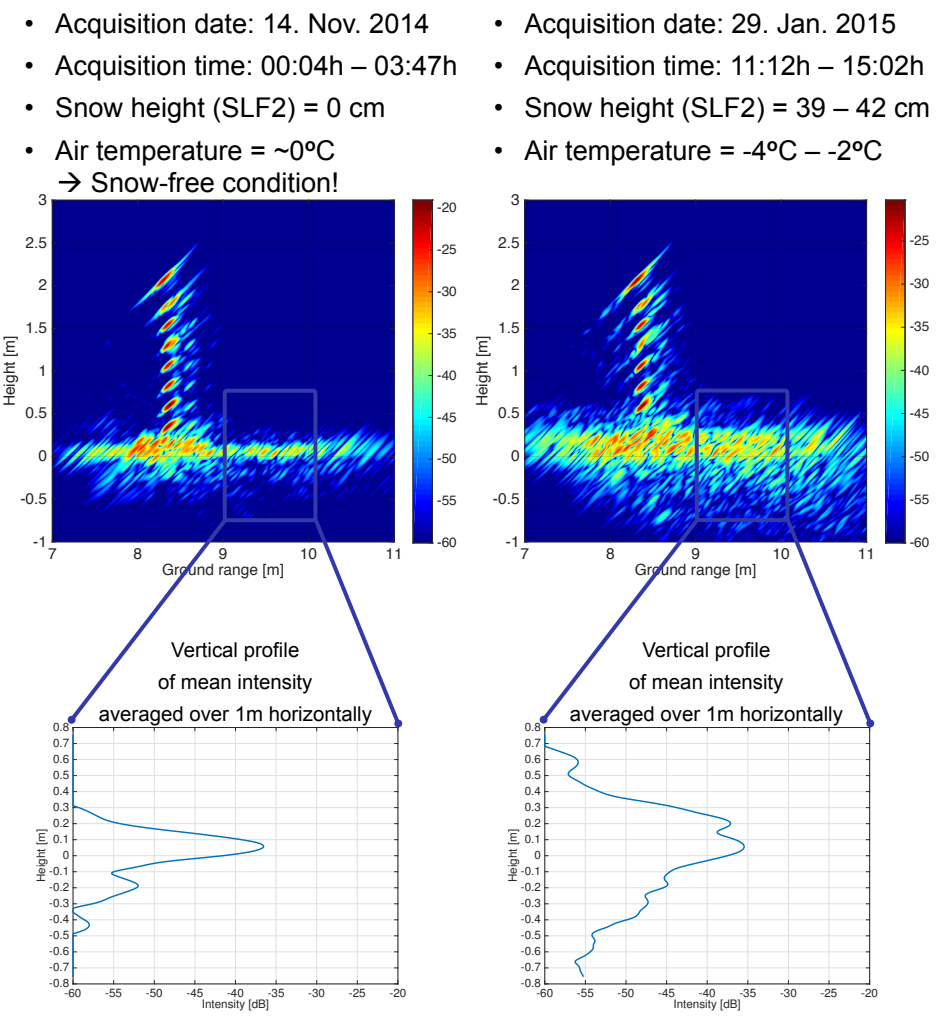

- Acquisition date: 01. Mar. 2015
- Acquisition time: $21: 02 \mathrm{~h}-23: 44 \mathrm{~h}$
- Snow height (SLF2) $=50-55 \mathrm{~cm}$
- Air temperature $=-1^{\circ} \mathrm{C}-0^{\circ} \mathrm{C}$
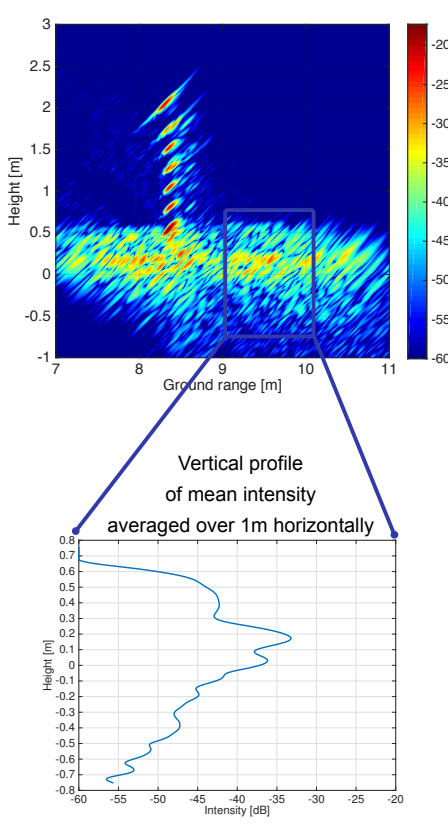

- Acquisition date: 03. Mar. 2015

- Acquisition time: 21:01h - 23:43h

- Snow height $(\mathrm{SLF} 2)=60-65 \mathrm{~cm}$

- Air temperature $=-4^{\circ} \mathrm{C}--^{\circ} \mathrm{C}$
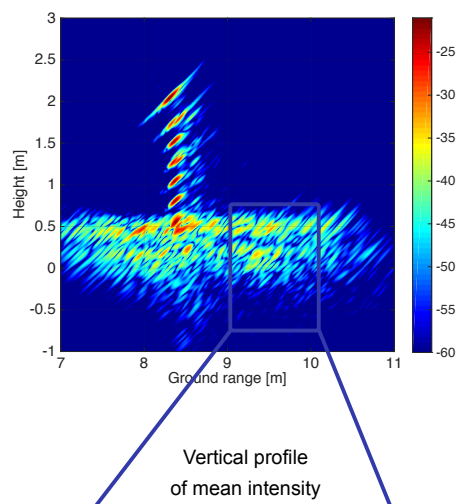

of mean intensity

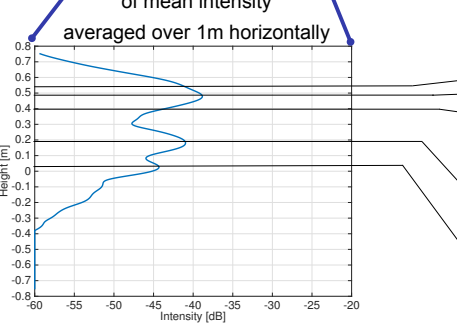

- Acquisition date: 15. Feb. 2015

- Acquisition time: 11:15h - 15:05h

- Snow height (SLF2) $=45-50 \mathrm{~cm}$

- Air temperature $=4.3^{\circ} \mathrm{C}-10.0^{\circ} \mathrm{C}$

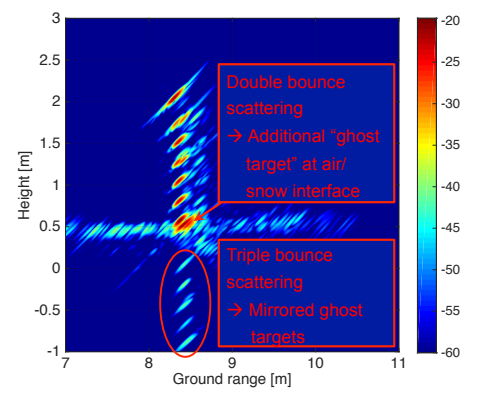

SnowMicroPen profile (left) and snow profile (right) 02. Mar. 2015 ("Rammsonde")

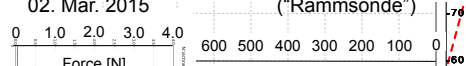
Force $[\mathrm{N}]$

Figure 2. Series of tomographic profiles (HH-channel) of the situation including the test target with its 8 aluminium spheres at the test site in Davos, Switzerland. Each 1-look image was obtained from 50 measurements along the rail. Aperture synthesis was performed by a time-domain back-projection approach taking into account a simple refraction model. Below each of the 2-D slices a vertical profile of mean intensity averaged over $1 \mathrm{~m}$ horizontally (see blue rectangle) is shown. Upper left: reference case under snow-free condition; only the surface of the meadow and the tomographic test target are visible. Upper right, lower left/middle: profile of the snowpack (and the tomographic test target) at different instances in time, temperature below melting point. The 1-D vertical profile of the case shown at the center of the lower row is also compared to in-situ snow profile measurement taken a day earlier. The comparison indicates that two of the three main melt-freeze crusts/ice layer as well as the ground surface are correctly resolved in the tomographic profile. Lower right: data acquisition under melted snow surface condition leading to double bounce and triple bounce (snow surface, sphere, snow surface) scattering which results in ghost targets in the tomographic image. 
snow profile measurements taken at the test site are displayed on the lower right of Fig. 2.

\section{DISCUSSION}

The new tomographic profiling observation capability of the enhanced SnowScat measurement setup is demonstrated by means of a tomographic test target and a first set of tomographic profiling measurements at a test site in Davos, Switzerland, under both snow-free and snowcovered conditions. The tomographic slices shown in Fig. 2, each of them obtained from $50 \mathrm{HH}$-polarized measurements along the rail, distinctly show (1) the specular reflection from the spheres of the test target, (2) the ground surface in snow-free conditions, and (3) different layers within the snowpack that were identified as meltfreeze crusts and ice layers in accompanying in-situ snow profile measurements. Tomographic profiles of average intensities, averaged along ground range, are in accordance with the most prominent horizontal layers of the snowpack as retrieved by means of the two in-situ snow profile measurements. The comparison indicates that two of the three main melt-freeze crusts/ice layer as well as the ground surface are correctly resolved in the tomographic profile. An interesting case (see Fig. 2, lower right) was also found when a tomographic measurement was taken under a melted snow surface condition where virtually no penetration into the snowpack, but instead, double and triple bounce scattering can be observed resulting in "ghost targets" of the spheres of the tomographic test target.

\section{CONCLUSION}

The new tomographic profiling capability of SnowScat now allows for investigating the complex electromagnetic interaction within snowpacks at a high spatial resolution at a dedicated test site, in a well-controlled environment, and over an extended period of time. This first test campaign has shown the high potential of the new tomographic profiling mode to resolve layered structures within a snowpack. Modeling of the refraction and the tomographic focusing need to be further refined to improve the tomographic imaging in the case where multiple layers are present in a thick snowpack. This aspect requires further investigation and testing with SnowScat tomographic profiling data of a more substantial snowpack.

\section{ACKNOWLEDGMENTS}

This work has been conducted in the frame of ESA/ESTEC Contract No. 20716/06/NL/EL CCN3, "Enhancement of SnowScat for tomographic and vertical profiling observation capabilities".

\section{REFERENCES}

1. A. Wiesmann, T. Strozzi, C. L. Werner, U. Wegmuller, and M. Santoro, "Microwave remote sensing of alpine snow," in Proc. IEEE Int. Geosci. Remote Sens. Symp., July 2007, pp. 1223-1227.

2. C. L. Werner, A. Wiesmann, T. Strozzi, M. Schneebeli, and C. Matzler, "The SnowScat ground-based polarimetric scatterometer: Calibration and initial measurements from Davos Switzerland," in Proc. IEEE Int. Geosci. Remote Sens. Symp., July 2010, pp. 23632366.

3. A. Wiesmann, C. L. Werner, T. Strozzi, C. Matzler, T. Nagler, H. Rott, M. Schneebeli, and U. Wegmuller, "SnowScat, X- to Ku-band scatterometer development," in Proc. ESA Living Planet Symposium, June 2010.

4. A. Wiesmann, C. Werner, C. Matzler, M. Schneebeli, T. Strozzi, and U. Wegmuller, "Mobile X- to Ku-band scatterometer in support of the CoRe-H2O mission," in Proc. IEEE Int. Geosci. Remote Sens. Symp., vol. 5, July 2008, pp. 244-247.

5. S. Tebaldini and L. Ferro-Famil, "High resolution three-dimensional imaging of a snowpack from ground-based SAR data acquired at X and Ku band," in Proc. IEEE Int. Geosci. Remote Sens. Symp., July 2013, pp. 77-80.

6. L. Ferro-Famil, S. Tebaldini, M. Davy, and F. Boute, "3D SAR imaging of the snowpack at X- and Ku-band: results from the AlpSAR campaign," in Proc. of EUSAR 2014 - 10th European Conference on Synthetic Aperture Radar, June 2014, pp. 1-4.

7. O. Frey, F. Morsdorf, and E. Meier, "Tomographic imaging of a forested area by airborne multi-baseline P-band SAR," Sensors, Special Issue on Synthetic Aperture Radar, vol. 8, no. 9, pp. 5884-5896, Sept. 2008.

8. O. Frey and E. Meier, "3-D time-domain SAR imaging of a forest using airborne multibaseline data at L- and P-bands," IEEE Trans. Geosci. Remote Sens., vol. 49, no. 10, pp. 3660-3664, Oct. 2011. 